\title{
Analysis of molecular variation in porcine reproductive and respiratory syndrome virus in China between 2007 and 2012
}

\author{
Yuhang Cao", Hongsheng Ouyang", Mingjun Zhang", Fuwang Chen, Xin Yang, Daxing Pang, Linzhu \\ Ren
}

Jilin Provincial Key Laboratory of Animal Embryo Engineering, College of Animal Sciences, Jilin University, Changchun 130062, Jilin, China

In the present study, 89 porcine reproductive and respiratory syndrome virus (PRRSV) isolates in China during 2007 to 2012 were randomly selected from the GenBank genetic sequence database. Evolutionary characteristics of these isolates were analyzed based on the sequences of non-structural protein 2 (Nsp2) and glycoprotein 5 (GP5). The genetic variations of the isolates were also compared with six representative strains. The results showed that a high degree of genetic diversity exists among the PRRSV population in China. Highly pathogenic PRRSV isolates, with a discontinuous deletion of a 30 amino acid residue in the Nsp2 region, remained the most dominant virus throughout 2007-2012 in China. Owing to the extensive use of representative vaccine strains, natural recombination events occurred between strains. Three isolates - HH08, DY, and YN-2011 - were more closely related to vaccine strains than the other isolates. Both YN-2011 and DY were the evolutionary products of recombination events between strains SP and $\mathrm{CH}-1 \mathrm{R}$. The results of the present study provide useful information for the epidemiology of PRRSV as well as for vaccine development.

KEYWORDS porcine reproductive and respiratory syndrome virus (PRRSV); open reading frame (ORF); non-structural protein 2 (Nsp2); glycoprotein 5 (GP5); recombination

\section{INTRODUCTION}

Porcine reproductive and respiratory syndrome (PRRS), caused by the porcine reproductive and respiratory syndrome virus (PRRSV), has been acknowledged as one of the most economically devastating diseases of the swine industry. The genome of PRRSV consists of approximately 15 kilobase pairs $(\mathrm{kb})$ and encodes nine overlapping open reading frames (ORFs), including non-structural protein (Nsp) coding sequence, and structural protein coding sequences ORF2 to ORF7 (Li B et al, 2010; Zhou Y et al, 2012). The non-structural protein, designated as polyprotein, can be divided into Nsp1 $\alpha$,

Received: 23 April 2014, Accepted: 10 June 2014

Published online: 17 June 2014

$\triangle$ Correspondence:

Phone: +86-431-87836175, Fax: +86-431-86758018,

Email: renlz@jlu.edu.cn

\#These authors contributed equally to this work.
Nsp1 $\beta$, and Nsp2-Nsp12 (Li B, et al, 2010; Music N, et al, 2010). ORFs 2-7, located upstream of the 3'-UTR (three prime untranslated region), encode viral structural proteins, such as glycoprotein 5 (GP5), glycoprotein 7 (GP7), and nucleocapsid protein (NP) (Li B et al, 2010; Music N et al, 2010).

It was reported that a so-called porcine "high fever" disease caused by highly pathogenic PRRSV (HP-PRRSV) emerged in the south of China in 2006, spreading to more than 10 provinces (autonomous cities or regions) and affecting over two million pigs, with about 400,000 fatal cases (Tian K, et al, 2007). However, research was lacking as to whether the HP-PRRSV isolates were dominant during 2007-2012. Considering the extensive use of PRRSV vaccines for preventing and controlling PRRS in mainland China, the appearance of a natural recombination event between a vaccine strain and a field isolate is noteworthy. In the present study, 89 PRRSV isolates in China during 2007 to 2012 were selected from the GenBank genetic sequence database. The evolutionary 
characteristics of these isolates were analyzed based on the sequences of Nsp2 and GP5. The genetic variations of the isolates were also compared with six representative strains. The results of the present study may prove useful for the epidemiology of PRRSV as well as for vaccine development.

\section{MATERIALS AND METHODS}

\section{Isolates}

In China, between 2007 and 2012, 89 PRRSV isolates were collected from the GenBank database (Supplementary Table 1). Six strains were selected as representative strains, including the representative American-type PRRSV vaccine strain VR2332, three vaccines strains used in China (CH-1R, MLV RespPRRS/Repro, and $\mathrm{SP}$ ), and two HP-PRRSV isolates (GD and JXA1). The ClustalW (multiple sequence alignment) program (Thompson J D, et al, 1994) was used to generate an alignment file for these PRRSV isolates.

\section{Phylogenetic analysis}

Phylogenetic and molecular evolutionary genetic analyses were conducted by using the distance-based neighbor-joining method with MEGA 5.1 software (Kumar S et al, 2004). Bootstrap values were calculated from 1,000 replicates of the alignment.

\section{Recombination analysis}

Recombination analysis was performed by comparing full-length genome sequences of the isolates using a recombination detection program (RDP), as described by Martin D P, et al (2010). Recombinant region and parental sequences were identified by five procedures (BootScan, Chimera, GENECONV, MaxChi, and RDP) integrated in an RDP4 software package, with correction for multiple comparisons $(\mathrm{P}<0.001)$.

\section{RESULTS}

\section{HP-PRRSV isolates were the dominant virus isolates during 2007-2012}

Nsp2 is the largest non-structural protein cleavage from a polyprotein (Snijder E J, et al, 1995; Zhang M, et al, 2013). Nsp2 has been recognized as the most variable region within the PRRSV genome (Allende R, et al, 1999; Yoshii M, et al, 2008; Zhou Y, et al, 2012; Wang F X, et al, 2013). The hypervariable region of Nsp2 in HP-PRRSV isolates contained a discontinuous deletion equivalent to 30 amino acids (Tian K, et al, 2007). Nsp2 is considered an important region for monitoring the genetic and epidemiological evolution of PRRSV (Wang F X, et al, 2013). In this study, the deduced amino acid (aa) sequences of 89 complete Nsp2 proteins of PRRSV exhibited different lengths, indicating that a great variety of PRRSV isolates exist in China (Supplementary Table 1). The length of amino acids ranged from 1022 aa to 1230 aa. There were 601 (48.9\%) variable sites, including singleton sites (13.1\%) and parsim-informative sites $(35.8 \%)$. These data suggested that a high degree of genetic diversity existed among the PRRSV population in China. Out of 89 Nsp2 proteins, $76(85.4 \%)$ had the same length of 1166 aa. Furthermore, 80 out of $89(88.9 \%)$ Nsp2 proteins had a discontinuous deletion of 30 amino acids in Nsp2, indicating that isolates with the discontinuous 30-aa deletion in the Nsp2 region represented the dominant virus during 2007-2012.

To explore the relationship between these isolates and six representative strains, a phylogenetic tree of the Nsp2 was constructed by using the distance-based neighbor-joining method with MEGA4 software. The results showed that the 89 isolates could be divided into three subgroups (Figure 1): subgroup I, II, and III. The majority of these isolates were grouped into subgroup I, whose representatives isolates were GD, JXA1, and vaccine strain CH-1R. Subgroup II was composed of GM, QYYZ, and QY2010. Finally, subgroup III was composed of DY, YN-2011, and three representative vaccine strains (VR2332, SP, and MLV RespPRRS/Repro). The sequence comparison showed that the Nsp2 of DY, YN2011, and HH08 had 83.61\%-99.67\%, 82.94\%-98.83\%, and $78.73 \%-98.49 \%$ homologous identity with that of vaccine strains at an amino acid level (Table 1). Isolates DY and YN-2011 had the greatest homology with MLV RespPRRS/Repro, while isolate HH08 exhibited the greatest homology with $\mathrm{CH}-1 \mathrm{R}$. Furthermore, comparing the Nsp2 aa sequence of DY with that of MLV RespPRRS/Repro, mutations were observed at position $\mathrm{T} 530 \rightarrow \mathrm{A} 530, \mathrm{E} 540 \rightarrow \mathrm{G} 540, \mathrm{~K} 569 \rightarrow \mathrm{E} 569$, and $\mathrm{K} 636 \rightarrow$ E636. Isolate HH08 had $98.49 \%$ homology to CH-1R, with 18 mutated sites in Nsp2. These results suggested that HP-PRRSV isolates represented the dominant virus during 2007-2012. Three isolates - HH08, DY, and YN-2011 - were more closely related to the vaccine strains.

\section{Recombination occurred due to extensive use of the representative vaccine strains}

Glycoprotein 5 (GP5) encoded by ORF5 is one of the major structural proteins. It comprises $2-4$ glycosylation sites, a 31-aa signal peptide, and 6 antigenic determinants that induce neutralizing antibodies (Yin G, et al, 2009; Zhou L, et al, 2009; Mu C, et al, 2013). GP5 is one of the structural proteins with the highest variability in PRRSV (Zhang M, et al, 2013). As the main candidate protein for the development of vaccine subunits, also as a result of its immunological significance and polymorphic nature, GP5 has been the target for analysis of the genetic diversity 


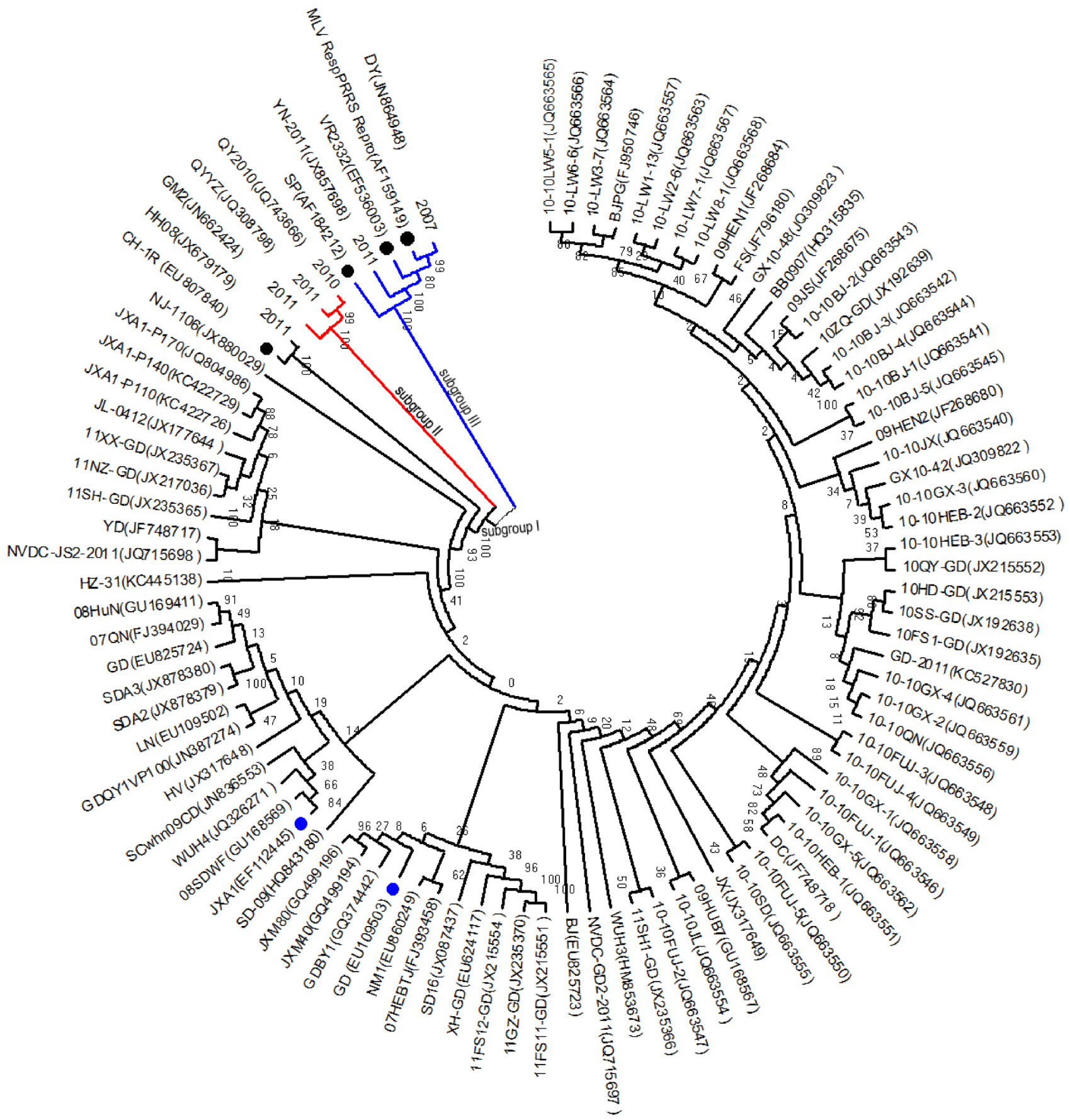

Figure 1. Phylogenetic tree representing 89 isolates during 2007-2012 and six representative strain sequences based on the Nsp2 genes. The phylogenetic tree was constructed by the distance-based neighbor-joining method using MEGA5.1 software. Bootstrap values were calculated from 1,000 replicates of the alignment. Different subgenotypes were indicated by different colors. Black branches indicate subgroup I, red branches indicate subgroup II, and blue branches indicate subgroup III. Six representative strains, including the representative American-type PRRSV vaccine strain VR2332 (EF536003), three vaccine strains used in $\mathrm{China}$ [CH-1R (EU807840), MLV RespPRRS/Repro (AF159149), and SP (AF184212)], and two HP-PRRSV isolates [GD (EU109503) and JXA1 (EF112445)], are indicated by filled circles. Black-filled circles indicate vaccine strains, while blue-filled circles indicate HP-PRRSV isolates. 
Table 1. Evolutionary divergence between sequences

\begin{tabular}{cccc}
\hline Species 1 & Species 2 & $\begin{array}{c}\text { Nsp2 } \\
\text { identity } \\
(\%)\end{array}$ & $\begin{array}{c}\text { ORF5 } \\
\text { identity } \\
(\%)\end{array}$ \\
\hline VR2332 & DY & 99.16 & 98.5 \\
\hline MLV RespPRRS/Repro & DY & $\underline{99.67}$ & $\underline{100}$ \\
\hline CH-1R & DY & 83.61 & 89 \\
SP & DY & 84.17 & 92 \\
VR2332 & YN-2011 & 98.49 & 97.5 \\
MLV RespPRRS/Repro & YN-2011 & $\underline{98.83}$ & $\underline{99}$ \\
SP & YN-2011 & 82.94 & 88 \\
VR2332 & YN-2011 & 83.77 & 91 \\
CH-1R & HH08 & 83.19 & 89 \\
SP & HH08 & 83.44 & 88.5 \\
\hline MLV RespPRRS/Repro & HH08 & $\underline{98.49}$ & $\underline{97.5}$ \\
\hline
\end{tabular}

* Underscored numbers indicate the highest identities between species 1 and 2 , respectively.

of PRRSV (Yin G, et al, 2009). The results in this study showed that the length of deduced amino acids of 89 complete ORF5 of PRRSV ranged from 199 aa to 201 aa (Supplementary Table 1). Out of 89 GP5 proteins, 86 had the same length of 200 amino acids (Supplementary Table 1). Isolate GD-2011 had 201 aa, with N60 insertion. Isolates 07QN, 09JS, and JX had 199 aa, with L200 deletion. There were $83(41.2 \%)$ variable sites, including singleton sites $(14.3 \%)$ and parsim-informative sites $(26.9 \%)$. The functional domains of GP5 - such as the signal peptide, ectodomain, transmembrane regions, endodomain, primary neutralizing epitope (PNE), and decoy epitope - were analyzed by Zhou Y et al (2009). Compared with the representative vaccine strains, the mutation sites of 89 isolates were located at position 37, 38, 39, 41, 43, and 52 of the PNE (Supplementary Table 1). The most variable site of the PNE was L39/F39, including L39/ $\mathrm{F} 39 \rightarrow \mathrm{S} 39$ (4 isolates) and $\mathrm{L} 39 / \mathrm{F} 39 \rightarrow \mathrm{I} 39$ (83 isolates). Other representative amino acid mutations of the PNE $(\mathrm{S} 37 \rightarrow \mathrm{P} 37, \mathrm{H} 38 \rightarrow \mathrm{Q} 38 / \mathrm{Y} 38, \mathrm{~L} 41 \rightarrow \mathrm{S} 41, \mathrm{Y} 43 \rightarrow \mathrm{C} 43$, and G52 $\rightarrow \mathrm{V} 52$ ) were also found in these 89 isolates. It has been reported that ORF5 codon positions 13 and 151 played a key role in denaturing VR2332 to MLV RespPRRSV (Yin G, et al, 2009). In this study, Q13 was observed in 7 of 89 isolates $(7.87 \%)$, while R13 was observed in the remaining 82 isolates (92.13\%) (Supplementary Table 2). A conserved G151 was observed in 89 isolates (Supplementary Table 2). Interestingly, the GP5 of isolates DY, YN-2011, and HH08 was highly homologous to four representative vaccine strains - VR2332, CH-1R, SP, and MLV
RespPRRS/Repro - with 89\%-100\%, 88\%-99\%, and $88.5 \%-97.5 \%$ identities, respectively (Table 1 ). Isolates DY and YN-2011 had the greatest homology with MLV RespPRRS/Repro, while isolate HH08 exhibited the greatest homology with $\mathrm{CH}-1 \mathrm{R}$. It could then be concluded that a recombination might have occurred between these strains.

\section{Extensive use of attenuated PRRSV vaccine contributed to the increasing diversity of PRRSV in the field}

Studies have shown that recombination events have occurred in PRRSV (van Vugt J, et al, 2001; Yuan S, et al, 1999; Liu D, et al, 2011; Lu W, et al, 2012; Chen N, et al, 2013; Shi M, et al, 2013). Lu W et al (2012) reported that isolate GM2 was a potential recombinant between the MLV RespPRRS/Repro vaccine strain and the Chinese field strain QYYZ. These events might have major implications for virus evolution, pathogenicity, vaccine safety and efficiency, or diagnosis. To confirm whether recombination occurred between these isolates, recombinant analysis was conducted based on the fulllength genome sequences of PRRSV isolates, using RDP4 software, as described by Martin D et al (2010). The results showed that the recombination sites were located in positions 3345-3707 and 12269-12981 (DY), or positions 3345-3707 and 12269-12955 (YN-2011) of the coding region of PRRSV isolates, with a significance level of $p<0.001$ (Figure 2). The recombinant fragment 3345-3707 was located in the Nsp2 coding region, while the recombinant fragment 12269-12981 (or 12269-12955) was located in the GP2 and GP3 coding regions. The strain SP was identified as the major parent, while the strain $\mathrm{CH}-1 \mathrm{R}$ was the minor parent (Figure 2 ). These results indicated that both isolates, namely YN-2011 and DY, were the evolutionary products of recombination events between $\mathrm{SP}$ and $\mathrm{CH}-1 \mathrm{R}$. It has been shown that recombination was an important genetic mechanism, contributing to the variation and evolution of PRRSV, and that HP-PRRSV was a variant of Type 2 PRRSV with high virulence (Liu D, et al, 2011; Chen N, et al, 2013). The results in this study further proved that extensive use of attenuated PRRSV vaccine also contributed to the increasing diversity of PRRSV in the field. In addition, mutations potentially correlated with the overattenuation of an HP-PRRSV strain (Yu X, et al, 2013). The antigenicity and immunogenicity of overattenuated vaccine were significantly reduced during the overattenuation (Yu X, et al, 2013). As recombination requires the co-infection of a pig with more than one PRRSV isolate, restrictions on usage of attenuated vaccine could help to reduce risks induced by recombination. Furthermore, the use of inactivated vaccines, which are currently available, is also problematic since they do not induce 


\begin{tabular}{|c|c|c|}
\hline \multirow[t]{2}{*}{ DY(JN864948) } & $3345-3707$ & $12269-12981$ \\
\hline & $\begin{array}{l}\mathrm{CH}-1 \mathrm{R}(\mathrm{EU} 807840) \\
\text { Major parent (similarity): SP }(93.7 \%) \\
\text { Minor parent (similarity): } \mathrm{CH}-1 \mathrm{R}(86.2 \%)\end{array}$ & $\begin{array}{l}\mathrm{CH}-1 \mathrm{R}(\mathrm{EU} 807840) \\
\text { Major parent (similarity): } \mathrm{SP}(93.8 \%) \\
\text { Minor parent (similarity): } \mathrm{CH}-1 \mathrm{R}(94.3 \%)\end{array}$ \\
\hline YN-2011(JX857698) & $3345-3707$ & $12269-12955$ \\
\hline & $\begin{array}{l}\mathrm{CH}-1 \mathrm{R}(\mathrm{EU} 807840) \\
\text { Major parent (similarity): } \mathrm{SP}(93.8 \%) \\
\text { Minor parent (similarity): } \mathrm{CH}-1 \mathrm{R}(86 \%)\end{array}$ & $\begin{array}{l}\mathrm{CH}-1 \mathrm{R}(\mathrm{EU} 807840) \\
\text { Major parent (similarity): } \mathrm{SP}(93.9 \%) \\
\text { Minor parent (similarity): } \mathrm{CH}-1 \mathrm{R}(93.9 \%)\end{array}$ \\
\hline
\end{tabular}

Figure 2. Recombination plots of the coding regions from selected sequences. Recombination analysis was performed by comparing full-length genome sequences of isolates DY, YN-2011, and HH08 with four vaccine strains (VR2332, CH-1R, SP, and MLV RespPRRS/Repro). Recombinant region and parental sequences were identified using five procedures (Bootscan, Chimera, GENECONV, MaxChi, and RDP) integrated in an RDP4 software package with correction for multiple comparisons $(P<0.001)$. Nucleotide positions of detected breakpoints are indicated.

effective protective immunity. We strongly recommend that priority should be given to the development and use of other vaccines, such as genetic vaccines and subunit vaccines. Moreover, the focus of attention should be directed toward measures to improve the immunogenicity of inactivated vaccine.

\section{DISCUSSION}

In summary, the results in this study have suggested that a high degree of genetic diversity has existed among the PRRSV population in China. HP-PRRSV isolates, with a discontinuous 30-aa deletion in Nsp2 region, were still the dominating virus during 2007-2012. Owing to the extensive use of the representative vaccine strains, recombination events occurred. Three isolates, namely HH08, DY and YN-2011, were more closely related to vaccine strains. Both the YN-2011 and DY isolates were the evolutionary products of recombination events between SP and CH-1R. The development and usage of attenuated vaccines for the disease should be reconsidered because of recombination between different isolates in the field.

\section{ACKNOWLEDGMENTS}

This work was supported by the Jilin Province Science and Technology Development Project (No. 20140101123 JC), the Fundamental Research Fund of Jilin University, and the Program for Changjiang Scholars and Innovative Research Team in University (No. IRT1248).

\section{COMPLIANCE WITH ETHICS GUIDELINES}

The authors declare that they have no conflicts of interest. This article does not contain any studies with hu- man or animal subjects performed by any of the authors.

\section{AUTHOR CONTRIBUTIONS}

RLZ designed the experiments. CYH performed the experiments. OYHS, ZMJ, CFW, and YX analyzed the data. RLZ and PD wrote the paper. All authors read and approved the final manuscript.

Supplementary tables are available on the website of Virologica Sinica: http://www.virosin.org.

\section{REFERENCES}

Allende R, Lewis T L, Lu Z, Rock D L, Kutish G F, Ali A, Doster A R, Osorio F A. 1999. North American and European porcine reproductive and respiratory syndrome viruses differ in nonstructural protein coding regions. J Gen Virol, 80: 307-315.

Chen N, Yu X, Wang L, Wu J, Zhou Z, Ni J, Li X, Zhai X, Tian K. 2013. Two natural recombinant highly pathogenic porcine reproductive and respiratory syndrome viruses with different pathogenicities. Virus Gen, 46: 473-478.

Kumar S, Tamura K, Nei M. 2004. MEGA3: integrated software for Molecular Evolutionary Genetics Analysis and sequence alignment. Brief Bioinform, 5: 150-163.

Li B, Fang L, Liu S, Zhao F, Jiang Y, He K, Chen H, Xiao S. 2010. The genomic diversity of Chinese porcine reproductive and respiratory syndrome virus isolates from 1996 to 2009. Vet Microbiol, 146: 226-237.

Liu D, Zhou R, Zhang J, Zhou L, Jiang Q, Guo X, Ge X, Yang H. 2011. Recombination analyses between two strains of porcine reproductive and respiratory syndrome virus in vivo. Virus Res, 155: 473-486.

Lu W, Wei Z, Zhang G, Li Z, Ma J, Xie Q, Sun B, Bi Y. 2012. Complete genome sequence of a novel variant porcine reproductive and respiratory syndrome virus (PRRSV) strain: evidence for recombination between vaccine and wild-type PRRSV strains. J Virol, 86: 9543.

Martin DP, Lemey P, Lott M, Moulton V, Posada D, Lefeuvre P. 2010. RDP3: a flexible and fast computer program for analyzing 
recombination. Bioinformatics, 26: 2462-2463.

Mu C, Lu X, Duan E, Chen J, Li W, Zhang F, Martin DP, Yang M, Xia P, Cui. 2013. Molecular evolution of porcine reproductive and respiratory syndrome virus isolates from central China. Res Vet Sci, 95: 908-912.

Music N, Gagnon C A. 2010. The role of porcine reproductive and respiratory syndrome (PRRS) virus structural and non-structural proteins in virus pathogenesis. Anim Health Res Rev, 11: 135-163.

Shi M, Holmes E C, Brar M S, Leung F C. 2013. Recombination is associated with an outbreak of novel highly pathogenic porcine reproductive and respiratory syndrome viruses in China. J Virol, 87: 10904-10907.

Snijder E J, Wassenaar A L, Spaan W J, Gorbalenya A E. 1995. The arterivirus Nsp2 protease. An unusual cysteine protease with primary structure similarities to both papain-like and chymotrypsin-like proteases. J Biol Chem, 270: 16671-16676.

Tian K, Yu X, Zhao T, Feng Y, Cao Z, Wang C, Hu Y, Chen X, Hu D, Tian X, Liu D, Zhang S, Deng X, Ding Y, Yang L, Zhang Y, Xiao H, Qiao M, Wang B, Hou L, Wang X, Yang X, Kang L, Sun M, Jin P, Wang S, Kitamura Y, Yan J, Gao G F. 2007. Emergence of fatal PRRSV variants: unparalleled outbreaks of atypical PRRS in China and molecular dissection of the unique hallmark. PLoS One, 2: e526.

Thompson J D, Higgins D G, Gibson T J. 1994. CLUSTAL W: improving the sensitivity of progressive multiple sequence alignment through sequence weighting, position-specific gap penalties and weight matrix choice. Nucleic Acids Res, 22: 4673-4680.

van Vugt J J, Storgaard T, Oleksiewicz M B, Bøtner A. 2001. High frequency RNA recombination in porcine reproductive and respiratory syndrome virus occurs preferentially between parental sequences with high similarity. J Gen Virol, 82: 26152620.

Wang F X, Song N, Chen L Z, Cheng S P, Wu H, Wen Y J. 2013. Non-structural protein 2 of the porcine reproductive and respiratory syndrome (PRRS) virus: a crucial protein in viral pathogenesis, immunity and diagnosis. Res Vet Sci, 95: 1-7.

Yin G, Gao L, Shu X, Yang G, Guo S, Li W. 2009. Genetic diversity of the ORF5 gene of porcine reproductive and respiratory syndrome virus isolates in southwest China from 2007 to 2009. PLoS One, 7: e33756.

Yoshii M, Okinaga T, Miyazaki A, Kato K, Ikeda H, Tsunemitsu H. 2008. Genetic polymorphism of the nsp2 gene in North American type--porcine reproductive and respiratory syndrome virus. Arch Virol, 153:1323-1334.

Yu X, Chen N, Deng X, Cao Z, Han W, Hu D, Wu J, Zhang S, Wang B, Gu X, Tian K. 2013. Genomic sequencing reveals mutations potentially related to the overattenuation of a highly pathogenic porcine reproductive and respiratory syndrome virus. Clin Vacci Immunol, 20: 613-619.

Yuan S, Nelsen C J, Murtaugh M P, Schmitt B J, Faaberg K S. 1999. Recombination between North American strains of porcine reproductive and respiratory syndrome virus. Virus Res, 61: 87-98.

Zhang M, Xie J, Sun L, Cao Z, Gu H, Deng S, Chen Y, Cao Z, Tang F, Su S, Zhang G. 2013. Phylogenetic analysis and molecular characteristics of 17 porcine reproductive and respiratory syndrome virus isolates in Southern China from 2010 to 2011. Microb Pathog, 65: 67-72.

Zhou L, Chen S, Zhang J, Zeng J, Guo X, Ge X, Zhang D, Yang H. 2009. Molecular variation analysis of porcine reproductive and respiratory syndrome virus in China. Virus Res, 145: 97-105.

Zhou Y, Yang X, Wang H N, Zhang A, Zhang Z, Kang R, Zeng F, Li H. 2012. Molecular characterization of a complete genome and 12 Nsp2 genes of PRRSV of southwestern China. Food EnvironVirol, 4: 102-114.

Zhou Y J, Yu H, Tian Z J, Li G X, Hao X F, Yan L P, Peng J M, An T Q, Xu A T, Wang Y X, Wei T C, Zhang S R, Cai X H, Feng L, Li X, Zhang G H, Zhou L J, Tong G Z. 2009. Genetic diversity of the ORF5 gene of porcine reproductive and respiratory syndrome virus isolates in China from 2006 to 2008. Virus Res, 144: $136-144$. 